\title{
Routing Problem with Multiple Transportation Modes Considering Road Damage
}

\author{
Weibo Zheng and Hong Zhou* \\ School of Economics and Management, Beihang University, Beijing 100191, China \\ ${ }^{*}$ Corresponding author
}

\begin{abstract}
A routing model with multiple transportation modes considering road damage is developed, and a two-stage variable neighborhood search algorithm is proposed for solving. The logistic function is used to describe the possible loss of a customer due to waiting, and the choice of transport mode depends on the trade-off between the cost of transportation and the waiting time of customers. Numerical experiments demonstrate that the proposed model can greatly reduce the total cost, compared with the traditional model which divides the demand nodes according to the information about the adjacency matrix. In addition, the algorithm proposed in this paper significantly outperforms the genetic algorithm in effectiveness.
\end{abstract}

Keywords-emergency logistics; multi-mode transportation; air-ground; road damage; VNS

\section{INTRODUCTION}

Disasters are usually unpredictable and accompanied with severe casualties (e.g., in 2004, the Boxing Day tsunami killed more than 200,000 people [1]; Wenchuan earthquake in 2008 caused more than 370,000 casualties [2]). Major disasters such as earthquakes often lead to road damage, water pollution, and lack of the necessities of life and medical treatment. Subsequent losses are closely related to the waiting time for emergency supplies, such as clean water and bandages, which are required to deliver to the affected areas in time. However, the deteriorated transportation situations due to road damage or block will increase the difficulties of rescue and result in even longer delivery time to the affected areas. To ensure the timeliness of rescue, it is a very important strategy to combine air and ground transportation in emergency logistics which has been studied by many researchers [3]. So far to the best of our knowledge, almost all the investigations on this problem divide customers (victims at different affected areas) into two categories based on different situations in advance, which is virtually to solve two independent routing problems. The method of how to divide the customers has a great influence on the optimization results.

In emergency logistics management, decision makers often balance waiting time of customers and rescue cost. HolguínVeras proposed a function to estimate the loss of waiting time [4], however the function they used would tend to be infinite when the waiting time is long enough which is not logical. In this paper, the logistic function was employed to estimate the loss due to waiting time. As the result, the customers were dynamically divided into two categories according to their waiting times. To solve this routing problem with multiple transportation modes (noted as MTMRP), a two-phase variable neighborhood search algorithm was proposed and experimented on an example composed of 80 nodes. Numerical experiments demonstrated that the proposed model can greatly reduce the total cost, compared with the traditional model which divides the customers (demand nodes) according to the information about adjacency matrix. Besides, the proposed algorithm also showed more superior performance than the genetic algorithm.

\section{PROBLEM Formulation}

\section{A. Problem Description}

The MTMRP under study in this paper considers road damage and the joint air-ground transportation which minimizes the total cost and loss due to waiting of customers. Two transportation tools, vehicles and helicopters, are available at each depot. The problem can be defined as follows.

Let $G=(N, A)$ be a directed graph, where $N=I \cup D$ is the set of nodes, where $I$ and $D$ represent the set of demand nodes and depots respectively, $A=\{(i, j) \in N \times N, i \neq j\}$ is the set of arcs. Each arc $(i, j) \in A$ is associated with two costs $c_{i j}^{K}$ and $c_{i j}^{H}$, which represent the travel time by vehicle and the flight time by helicopter, respectively. The loss due to waiting is defined by the logistic function $d P / d t=r P(E-P) / E$, and can be solved as

$$
P(t)=\frac{E P_{0}}{P_{0}+\left(E-P_{0}\right) \exp (-r t)}
$$

where $t$ is waiting time, $E$ is the maximum loss of waiting time, $P_{0}$ is the initial value at $t=0\left(P_{0} \neq 0\right)$, and $r$ is the rate of growth. For the convenience of processing, set $P_{0}=1$ when $E$ is big enough. Suppose that $T$ is the inflexion point of the function, it is easy to conclude that $r=\ln (E-1) / T$.

\section{B. Assumptions}

- $\quad$ Each demand node must be visited once and only once.

- Each route must starts and ends at the same depot.

- $\quad$ Each depot has sufficient supplies.

- The total demand for all customers on one route cannot exceed the capacity of a vehicle or helicopter. 
- The travel time by vehicle depends on the road condition, represented with the road unimpeded coefficient.

- The loading and service time can be ignored.

\section{Notation Definition}

Sets

I Set of demand nodes

$D$ Set of depots

$K$ Set of vehicles

$H$ Set of helicopters

\section{Parameters}

$K_{t} \quad$ The travel cost per time unit of a vehicle

$H_{t} \quad$ The flight cost per time unit of a helicopter

$K_{m}$ The fixed cost of each vehicle

$H_{m}$ The fixed cost of each helicopter

$c_{i j}^{K} \quad$ The travel time of a vehicle from node $i$ to $j$

$c_{i j}^{H} \quad$ The flight time of a helicopter from node $i$ to $j$

$\lambda_{i j}$ Road unimpeded coefficient, belonged to $(0,1]$

$q_{i} \quad$ The demand of node $i$

$Q^{c}$ The capacity of the conveyance $c$

$M$ A big enough positive constant

E Maximum loss of waiting time

$r$ The rate of growth for the logistic function

\section{Decision Variables}

$x_{i j}^{c} 1$ if the conveyance $c$ visits node $j$ immediately after $i$; 0 otherwise

\section{Other Variables}

$t_{i}^{c} \quad$ The arrival time of the conveyance $c$ at the node $i$

$n^{c} \quad$ The number of nodes serviced by the conveyance $c$

\section{Mathematical Model}

The objective to be minimized includes three components, the weighted total loss of waiting time (suppose that the importance of a demand node can be characterized by its demand $q_{i}$ ), the total fixed cost for conveyance, and the total travel cost, noted as $F_{1}, F_{2}$, and $F_{3}$, respectively.

$$
\begin{gathered}
F_{1}=\sum_{i \in I} q_{i} \frac{E}{1+(E-1) \exp \left(-r t_{i}\right)} \\
F_{2}=K_{m} \sum_{k \in K} \sum_{d \in D} \sum_{i \in I} x_{d i}^{k}+H_{m} \sum_{h \in H} \sum_{d \in D} \sum_{i \in I} x_{d i}^{h}
\end{gathered}
$$

$$
F_{3}=K_{t} \sum_{k \in K} \sum_{i \in I \cup D} \sum_{j \in I \cup D} x_{i j}^{k} c_{i j}^{K}+H_{t} \sum_{h \in H} \sum_{i \in I \cup D} \sum_{j \in I \cup D} x_{i j}^{h} c_{i j}^{H}
$$

Thus the model can be formulated as

Minimize:

$$
F_{1}+F_{2}+F_{3}
$$

s.t.

$$
t_{i}+c_{i j}^{K} / \lambda_{i j}-\left(1-\sum_{k \in K} x_{i j}^{k}\right) M \leq t_{j}, \quad \forall i \in I \cup D, \forall j \in I, i \neq j
$$

$$
t_{i}+c_{i j}^{H}-\left(1-\sum_{h \in H} x_{i j}^{h}\right) M \leq t_{j}, \forall i \in I \cup D, \forall j \in I, i \neq j
$$

$$
\sum_{j \in I \cup D} x_{i j}^{c}=\sum_{j \in I \cup D} x_{j i}^{c} \quad \forall i \in I, \quad \forall c \in K \cup H
$$

$$
\sum_{c \in K \cup H} \sum_{\substack{j \in I \cup D \\ i \neq j}} x_{i j}^{c}=1 \quad \forall i \in I
$$

$$
\sum_{i \in I \cup D} \sum_{j \in I \cup D} x_{i j}^{c}=n^{c}+1 \quad \forall c \in K \cup H
$$

$$
\begin{gathered}
\sum_{i \in I \cup D} \sum_{j \in I} x_{i j}^{c} q_{j} \leq Q^{c} \quad \forall c \in K \cup H \\
\sum_{i \in I} x_{d i}^{c}=\sum_{i \in I} x_{i d}^{c} \quad \forall c \in K \cup H, \forall d \in D
\end{gathered}
$$

$$
t_{i} \geq 0 \quad \forall i \in I \cup D
$$

$$
x_{i j}^{c} \in\{0,1\} \quad \forall i, j \in I \cup D, \quad \forall c \in K \cup H
$$

In above model, all conveyances are supposed to start at time 0 , so the waiting time of each node is just the arrival time and can be calculated via constraints (6) and (7). Constraints (8) and (9) ensure each demand node being visited exactly once. Constraint (10) is used to eliminate subtours. Constraint (11) sets the restriction on the shipment of conveyances. Constraint (12) assures that a route begins and ends at the same depot.

\section{VARIable NeIghborhoOd SEARCH Algorithm}

A VNS algorithm is proposed in this section which consists of two phases. In the first phase, an encoded initial solution is 
randomly created. Four heuristics according to the variable neighborhood descent (VND) strategy of Nenad and Hansen[5] are implemented until no further improvements can be achieved. Considering that the solution which makes the vehicle as full as possible is generally not optimal, a further improvement procedure is conducted in the second phase based on the previously resulted solution. First, decode the best solution obtained in first phase. Then seven different heuristics based on the VND strategy are attempted.

\section{A. Solution Representation}

In this algorithm, a solution is encoded into several segments which represent the routes of all assigned vehicles and helicopters. The $k$ th segment starts from the first demand node served by the $k$ th vehicle and stop at the node where the $k$ th vehicle has not enough capacity to serve the next node. The depot which is closest to the first or last node of the segment is selected as the source node of that route. The number 0 is used to separate the routes for the two different conveyances, vehicles before 0 and helicopters after 0 .

An example for the solution is shown in Figure 1. The route of the first vehicle starts from node 3 . If the total demand of node 3, 2, 6 exceeds the vehicle capacity, the route will end at node 2 . The route of the second vehicle starts from node 6 , and end at node 1 . Then turn to the air transportation mode after the symbol 0 . The route of the first helicopter starts from node 4 , and end at node 5 .

\begin{tabular}{|l|l|l|l|l|l|l|}
3 & 2 & 6 & 1 & 0 & 4 & 5 \\
\hline
\end{tabular}

FIGURE I. EXAMPLE OF A FEASIBLE SOLUTION FOR THE MTMRP.

When decoding the solution, a proper depot has to be assigned to each of the routes. Suppose according to the above assignment criterion (closest to the route), depot $d_{1}$ is assigned to the route $6-1$, depot $d_{2}$ is assigned to the route $3-2$ and $4-5$. Then the decoded solution is shown in Figure 2 and Figure 3.

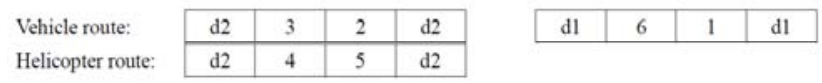

FIGURE II. THE DECODED SOLUTION.

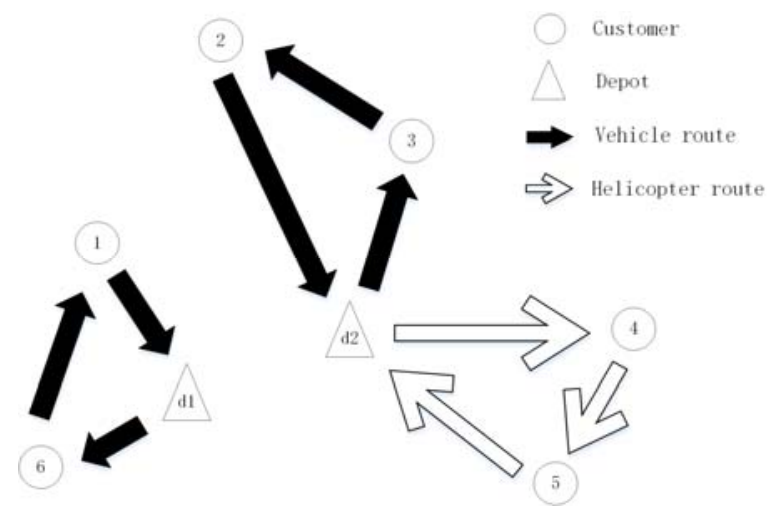

FIGURE III. ROUTES OF THE SOLUTION

\section{B. The First Phase Heuristics}

Four heuristics are employed in this phase: 2-Opt, Or-Opt, 3-Opt, and SHIFT operator. These operators are "intra-route", and operate on the single route.

Suppose $i, j, k$ are three positions in a solution, $i<j<k$. The 2-Opt heuristic first opens the $\operatorname{arc}(i-1, i)$ and the $\operatorname{arc}(k, k+1)$; then reverse the route $i \sim k$; and finally connect the arc $(i-1, k)$ and the arc $(i, k+1)$. The process iterates until no further improvement is found. The Or-Opt heuristic opens arcs $(i-1, i),(j-1, j),(k, k+1)$ and then connect arcs $(i-1, j)$, $(k, i),(j-1, k+1)$. Correspondingly, The 3-Opt heuristic opens 3 arcs, however, there are 3 connections. SHIFT operator shifts the symbol 0 until no further improvement is achieved.

These procedures are implemented in accordance with the VND scheme: SHIFT is first applied till no further improvement can be found; then 2-Opt is performed till no further improvement, followed by Or-Opt; finally 3-Opt is applied in the same manner. If any of the heuristics succeeds in improving the route, the algorithm repeats again with SHIFT.

\section{The Second Phase Heuristics}

There are seven heuristics adopted in the second phase. Three of them are 2-Opt, Or-Opt and 3-Opt introduced in the first phase. The remaining four are "inter-route" operations: EXCHANGE, RELOCATE, 2-Opt*, and CROSS-exchange.

The RELOCATE operation inserts a single node in another route. The EXCHANGE heuristic swaps the position of two nodes in two different routes. The 2-Opt* choose two different routes, then opens an arc for each route, and swap the route after the breakpoint. The CROSS-exchange swaps two segments of nodes for two different routes.

The VND scheme for the four inter-route heuristics is: RELOCATE is first applied until no further improvements; then EXCHANGE, 2-Opt*, and CROSS-exchange. If any improvement is achieved by inter-route VND procedure, attempt the intra-route VND in such order as 2-Opt, Or-Opt and 3-Opt. The algorithm ends if no more improvement can be made.

\section{NUMERICAL EXPERIMENT}

There are 80 nodes randomly generated, among which 3 are depots and all the others are demand nodes. The demand for each node is an integer randomly generated between 1 and 5 (ton), and the unimpeded coefficient for each road is randomly generated from a beta distribution.

The flight time between any two nodes is calculated by Euclidean distance. The travel time for vehicles between two connected nodes is calculated by Euclidean distance with road unimpeded coefficient, while that between two nodes without connected road is calculated by Dijkstra algorithm. Thus the adjacency matrix of the travel time can be obtained. Parameters for the model are given in Table 1 . 
TABLE I. PARAMETERS FOR THE PROBLEM

\begin{tabular}{|l|l|c|c|c|c|}
\hline & $\begin{array}{c}\text { Fixed } \\
\text { cost (\$) }\end{array}$ & $\begin{array}{c}\text { Cost per } \\
\text { hour (\$) }\end{array}$ & $\begin{array}{c}\text { Capaci } \\
\text { ty (t) }\end{array}$ & $\begin{array}{c}\text { Maximum } \\
\text { loss (\$) }\end{array}$ & $\begin{array}{c}\text { Inflexion } \\
\text { point (h) }\end{array}$ \\
\hline Vehicle & 1,000 & 250 & 20 & \multirow{2}{*}{100,000} & 10 \\
\hline Helicopter & 50,000 & 10,000 & 25 & & \\
\hline
\end{tabular}

The above two phase VNS algorithm, was implemented in MATLAB and tested on a computer with Intel Xeon E3 (8 GB RAM). The evolution of the best solution in Figure 4 shows a satisfied convergence of the proposed algorithm.

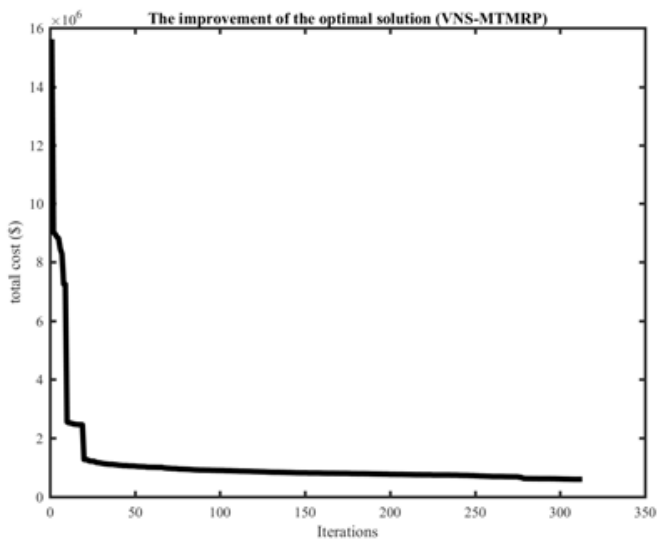

FIGURE IV. THE IMPROVEMENT OF THE OPTIMAL SOLUTION FOR VNS-MTMRP

A variety of genetic algorithms for multi-depot VRP were reviewed by Sašo and Podgorelec [6]. We chose the commonly used one with OX crossover, SWP mutation and tournament selection scheme to compare with our algorithm. In order to analyze the significance of the loss due to waiting, we also compared the results with and without the objective $F_{1}$, denoted as MTMRP and MTMRP2, respectively. The quality of the solution achieved in the similar running time was adopted as the main evaluation criterion.

TABLE II. COMPUTATIONAL RESULTS

\begin{tabular}{|l|c|c|c|}
\hline & $\begin{array}{c}\text { VNS- } \\
\text { MTMRP }\end{array}$ & $\begin{array}{c}\text { VNS- } \\
\text { MTMRP2 }\end{array}$ & $\begin{array}{c}\text { GA- } \\
\text { MTMRP }\end{array}$ \\
\hline First phase best solution (\$) & $5.86 \mathrm{E} 05$ & $5.70 \mathrm{E} 04$ & $9.87 \mathrm{E} 05$ \\
\hline Second phase best solution (\$) & $5.42 \mathrm{E} 05$ & $5.69 \mathrm{E} 04$ & $7.13 \mathrm{E} 05$ \\
\hline Improvement rate against GA & $7.52 \%$ & $0.21 \%$ & $27.8 \%$ \\
\hline Average waiting time (h) & 0.51 & 2.3 & 0.72 \\
\hline Transportation costs (\$) & $5.42 \mathrm{E} 05$ & $5.69 \mathrm{E} 04$ & $6.986 \mathrm{E} 05$ \\
\hline Loss of waiting time (\$) & $4.24 \mathrm{E} 03$ & $4.92 \mathrm{E} 06$ & $1.44 \mathrm{E} 04$ \\
\hline Total cost (\$) & $5.42 \mathrm{E} 05$ & $4.97 \mathrm{E} 06$ & $7.13 \mathrm{E} 05$ \\
\hline CPU time (s) & $2.29 \mathrm{E} 03$ & $2.47 \mathrm{E} 03$ & $2.50 \mathrm{E} 03$ \\
\hline
\end{tabular}

As shown in Table 2, the solution obtained by the proposed VNS is significantly superior to that by GA (the operation in the second phase for GA is the same as VNS). The average waiting time for MTMRP is significantly lower than MTMRP2 which implies that the rescue might be severely delayed without optimizing the loss of waiting time. In fact, there is no helicopter assigned in the solution to MTMRP2, which also explains the result. Although transportation cost can be reduced for MTMRP2, the possible loss due to delay in rescue may be fatal.

To demonstrate the advantage of our model over the traditional methods which divide customers into two fixed categories in advance, we compare our results with the method by Yang et al [7]. When the road unimpeded coefficient is lower than the threshold, the demand of customers will be fulfilled by the helicopter.

TABLE III. THE OPTIMAL SOLUTION UNDER DIFFERENT THRESHOLD

\begin{tabular}{|c|c|c|c|c|c|}
\hline & \multicolumn{5}{|c|}{ Threshold } \\
\cline { 2 - 6 } & 1 & 0.95 & 0.9 & 0.85 & 0.8 \\
\hline $\begin{array}{c}\text { Optimal } \\
\text { solution (\$) }\end{array}$ & $7.64 \mathrm{E} 5$ & $7.29 \mathrm{E} 05$ & $9.00 \mathrm{E} 05$ & $1.08 \mathrm{E} 06$ & $1.97 \mathrm{E} 6$ \\
\hline $\begin{array}{c}\text { CPU time } \\
\text { (s) }\end{array}$ & 35.43 & 26.57 & 15.85 & 15.31 & 11.89 \\
\hline
\end{tabular}

As shown in Table 3, the method of categorizing in advance can greatly reduce the search time, however the quality of solution is significantly influenced.

\section{CONCLUSIONS}

In this paper, a mixed air-ground transportation routing model is developed for the problem of emergency logistics with road damage. A two phase VNS is proposed to solve the problem. From the experimental results, it can be concluded that the consideration for integrating the loss due to waiting into optimization objective is quite essential for rescue decisions. In addition, it can achieve a better performance in solution to divide customers dynamically based on the road status, compared with the methods dividing the customers into fixed categories in advance.

\section{ACKNOWLEDGMENT}

This work is supported by the Natural Science Foundation of China under Grant No.71471007.

\section{REFERENCES}

[1] P. Dias, R. Dissanayake, and R. Chandratilake, "Lessons learned from tsunami damage in Sri Lanka," P. I. Civil Eng.-Civ. En. London, vol. 159, pp. 74-81, Thomas Telford Ltd, 2006.

[2] L Zhang,X Liu,Y Li, et al. "Emergency medical rescue efforts after a major earthquake: lessons from the 2008 Wenchuan earthquake," Lancet, England, vol. 379, pp. 853-861, March 2012.

[3] M. Zhang, J. Yu, Y. Zhang, and H. Yu, "Programming model of emergency scheduling with combined air-ground transportation," Adv. Mech. Eng. vol. 9, p. 1687814017739512 , November 2017.

[4] J. Holguín-Veras, , P. Noel, J. Miguel, L. N. Van Wassenhove, and F. Aros-Vera, "On the appropriate objective function for post-disaster humanitarian logistics models," J. Oper. Manag. Netherlands, vol. 31, pp. 262-280, Jul 2013.

[5] M. Nenad, and P. Hansen. "Variable neighborhood search," Comput. oper. res. England, vol. 24, pp. 1097-1100, 1997.

[6] K. Sašo, and V. Podgorelec, "A survey of genetic algorithms for solving multi depot vehicle routing problem," Appl. Soft Comput. Netherlands, vol. 27 pp. 519-532, 2015.

[7] X. Yang, Y. Jiang, Y. Zhang, and Z. Liu, "Vehicle type and route selection for emergency logistic management under road damage," IEEE Intern. Conf. Ser. Oper. Log. \& Inform, pp. 100-105, 2015. 